\title{
Deep Learning for Map Generalization: Towards a new Approach using Vector Data
}

\author{
Martin Knura ${ }^{\text {a }}$ \\ ${ }^{a}$ Lab for Geoinformation and Geovisualization (g2lab), HafenCity University Hamburg, Germany, martin.knura@hcu-hamburg.de
}

Keywords: Deep Learning, Map Generalization, Vector Data, Building Generalization

\begin{abstract}
:
Over the last decades, the research topic of automated map generalization was subject to several paradigm shifts, introducing new approaches and techniques like constraints (Harrie \& Weibel 2007) and agent-based modeling (Duchêne et al. 2018) to cartography. As with the paradigms before, the rising complexity of handling the current approach is about to trigger a new paradigm shift in automated map generalization. Due to the ongoing success in applications like Computer Vision, Speech Detection or Natural Language Processing, deep learning is a promising candidate to be the next paradigm in map generalization (Touya et al. 2019).
\end{abstract}

First experiments with deep learning applications in the field of map generalization, focusing on building generalization (Feng et al. 2019), mountain road generalization (Courtial et al. 2020), and data enrichment (Touya \& Lokhat 2020), confirm the potential of this approach to a certain degree. Nevertheless, all these experiments adapt models from image processing, creating input data by rasterizing geographical vector data. While the benefit of using the well-adapted deep learning models for image processing is undoubted, using only derived images as input comes along with a clear limitation in data richness and information compared to the underlying vector data (Touya et al. 2019), as well as with introducing uncertainty and fuzziness into the generalization process through rasterization (Liao et al. 2012).

By contrast, using vector-data as input would skip the rasterization process with all its problems, with the potential to preserve parts of the well-established workflow of map generalization for further editing. In all of the aforementioned experiments focusing on map generalization, the definition of the loss function was a limiting factor for the performance of the models and identified by the authors as primary subject for further research. Generating vector data as the output would not only avoid most of the problems resulting from pixel-based loss measures, but also offer opportunities to perform geometric calculations and generalization operations as part of more advanced and efficient loss functions.

Therefore, this dissertation project aims at developing a deep learning model for map generalization based on vector data. Currently, three deep learning approaches are under consideration: recurrent neural networks, graph convolutional neural networks and deep reinforcement learning. Recurrent neural networks process input data sequentially, and are able to work with geospatial vector polygons (van 't Veer et al. 2019). As a more general variant of CNNs, graph convolutional neural networks are also able to use vector data as input (Yan et al. 2019), and have the potential to perform similar tasks as applied in the experiments with building and mountain road generalizations. Deep Reinforcement Learning (Arulkumaran et al. 2017) on the other hand offers the possibility to integrate findings of former map generalization research into the learning process, e.g. using the fulfilling of constraints as rewards.

The presentation will provide an overview of the current state of the project, discuss the challenges and preliminary findings of first experiments with recurrent neural networks, and will outline future work.

\section{References:}

Arulkumaran, K., Deisenroth, M. P., Brundage M. \& Bharath, A. A. (2017): Deep Reinforcement Learning: A Brief Survey. IEEE Signal Processing Magazine 34 (6): 26-38. DOI: https://doi.org/10.1109/MSP.2017.2743240

Courtial, A., El Ayedi, A., Touya, G. \& Zhang, X. (2020): Exploring the Potential of Deep Learning Segmentation for Mountain Roads Generalisation. ISPRS Int. J. Geo-Inf. 2020, 9(5), 338; DOI: https://doi.org/10.3390/ijgi9050338

Duchêne, C., Touya, G., Taillandier, P., Gaffuri, J., Ruas, A., \& Renard, J. (2018): Multi-agents systems for cartographic generalization: Feedback from past and on-going research (Technical report). France: IGN. Internet: https://hal.archives-ouvertes.fr/hal-01682131

Feng, Y., Thiemann, F. \& Sester, M. (2019): Learning Cartographic Building Generalization with Deep Convolutional Neural Networks. ISPRS Int. J. Geo-Inf. 2019, 8(6), 258; DOI: https://doi.org/10.3390/ijgi8060258 
Harrie, L. \& Weibel, R. (2007): Modelling the Overall Process of Generalization. In: Mackaness, W., Ruas, A. \& Sarjakoski, L.T. (Ed.): Generalisation of Geographic Information: Cartographic Modelling and Applications. Elsevier: 67-87. DOI: https://doi.org/10.1016/B978-0-08-045374-3.X5000-5

Liao, S., Bai, Z. \& Bai, Y. (2012): Errors prediction for vector-to-raster conversion based on map load and cell size. Chin. Geogr. Sci. 22: 695-704. DOI: https://doi.org/10.1007/s11769-012-0544-y

Touya, G. \& Lokhat, I. (2020): Deep Learning for Enrichment of Vector Spatial Databases: Application to Highway Interchange. ACM Trans. Spatial Algorithms Syst. 6 (3): 21. DOI: https://doi.org/10.1145/3382080

Touya, G., Zhang, X. \& Lokhat, I. (2019): Is deep learning the new agent for map generalization? International Journal of Cartography, 5(2-3), 142-157; DOI: https://doi.org/10.1080/23729333.2019.1613071

Van 't Veer, R., Bloem, P. \& Folmer, E. (2019): Deep Learning for Classification Tasks on Geospatial Vector Polygons. Preprint: https://arxiv.org/abs/1806.03857v2

Yan, X., Ai, T., Yang, M. \& Yin, H. (2019): A graph convolutional neural network for classification of building patterns using spatial vector data. ISPRS Journal of Photogrammetry and Remote Sensing 150, 259-273; DOI: https://doi.org/10.1016/j.isprsjprs.2019.02.010 